\title{
Loss of claudin-7 is a negative prognostic factor for invasion and metastasis in oral squamous cell carcinoma
}

\author{
KUNIO YOSHIZAWA ${ }^{1}$, SHINICHI NOZAKI ${ }^{2}$, AKI KATO ${ }^{1}$, MARIKO HIRAI ${ }^{1}$, MIZUKI YANASE ${ }^{1}$, \\ TAISUKE YOSHIMOTO ${ }^{1}$, IYO KIMURA ${ }^{1}$, SHIRO SUGIURA ${ }^{1}$, AYAKO OKAMUNE ${ }^{1}$, HIROKO KITAHARA ${ }^{1}$, \\ NATSUYO NOGUCHI ${ }^{1}$, KOROKU KATO ${ }^{1}$, KOICHIRO UEKI ${ }^{1}$ and SHUICHI KAWASHIRI ${ }^{1}$ \\ ${ }^{1}$ Department of Oral and Maxillofacial Surgery, Kanazawa University Graduate School of Medical Science, \\ Kanazawa, Ishikawa 920-8641; ${ }^{2}$ Department of Dentistry and Oral Surgery, National Hospital \\ Organization, Kanazawa Medical Center, Kanazawa, Ishikawa 920-8650, Japan
}

Received August 30, 2012; Accepted November 2, 2012

DOI: $10.3892 /$ or.2012.2161

\begin{abstract}
Claudin-7 belongs to the claudin family, which consists of 24 subtypes of essential tight junction (TJ) integral membrane proteins with molecular weights of 20-27 kDa. We investigated the interrelationship between clinicopathological findings and claudin-7 expression in oral squamous cell carcinoma (OSCC). Using immunohistochemical techniques to examine the expression levels of claudin-7 in 67 cases of OSCC, claudin-7 expression was detected in 35 (52.2\%) of the 67 cases. We also compared the clinicopathological features of the OSCC cases with claudin-7 expression levels. Moreover, six cell lines with various invasive properties were investigated in vitro to compare mRNA and protein levels of claudin-7 using reverse transcription-polymerase chain reaction (RT-PCR) and the western blotting method. Decreased claudin-7 expression correlated significantly with T-category $(\mathrm{p}<0.05)$, lymph node metastasis $(p<0.01)$, and mode of invasion $(p<0.001)$. Patients with positive claudin-7 expression had a significantly better prognosis $(\mathrm{p}<0.05)$. Claudin-7 protein and mRNA levels were lower in the HOC313 and TSU cells, which have higher invasive potentials compared with other cell lines. These results suggest that loss of claudin-7 expression is associated closely with invasion and lymph metastasis and is an unfavorable prognostic factor in patients with OSCC.
\end{abstract}

Correspondence to: Dr Kunio Yoshizawa, Department of Oral and Maxillofacial Surgery, Kanazawa University Graduate School of Medical Science, 13-1 Takaramachi, Kanazawa, Ishikawa 920-8641, Japan

E-mail: yoshizawa@oral.m.kanazawa-u.ac.jp

Abbreviations: OSCC, oral squamous cell carcinoma; RT-PCR, reverse transcription-polymerase chain reaction; TJ, tight junction; UICC, Union International Contre le Cancer; LSAB, labeled streptavidin-biotin; EMT, epithelial-mesenchymal transition

Key words: carcinoma, tight junctions, prognosis, epithelial to mesenchymal transition

\section{Introduction}

Claudin family consists of 24 subtypes of essential tight junction (TJ) integral membrane proteins that have molecular weights of 20-27 kDa and contain four transmembrane domains (1-3). Tight junctions are responsible for the formation and maintenance of the permeability barrier in polarized epithelial cells. The gene has subsequently been localized to epithelial cells in a variety of tissues, including oral squamous epithelium, and its protein shows tissue-specific distribution patterns (4-6). It has become clear that, in addition to having tissue- and cell-specific features, modification or loss of these dynamic structures contributes to cancerization (7-10). However, how the role of claudin-7 contributes to cancerization in squamous cell carcinoma remains to be elucidated, and the patterns of claudin-7 expression in carcinoma vary as shown below $(3,11-18)$. Loss of claudin-7 has been reported to correlate with a poor prognosis in esophageal, colorectal, and nasopharyngeal cancers $(3,11-14)$. On other hand, upregulation of claudin-7 has been reported to correlate with poor prognosis of carcinogenesis in ovarian, breast, and gastric carcinomas (15-18).

The TNM classification which was proposed by the Union Internationale Contre le Cancer (UICC) (19) is a good system for describing the condition of cancer patients. However, this system cannot predict the biological characteristics of tumor cells. It is there therefore important to look for new objective prognostic factors that provide additional information on the biological characteristics of tumors. It is believed that invasion and metastasis are the most crucial characteristics of malignant tumors. Thus, mode of invasion is used as a histopathological classification category in oral squamous cell carcinoma (OSCC), as described by Yamamoto et al (20), and this classification is frequently used to predict progression, metastasis and prognosis (20-25) (Table I). To provide proper treatment, it is also important to examine the characteristics of cancer cells at the invasive front of OSCC.

We examined immunohistochemically the expression of claudin-7 in vivo and compared its expression in cell lines derived from invasive OSCC in vitro to investigate the interrelationship between clinicopathological factors including 
criteria on mode of invasion and claudin-7 expression in OSCC.

\section{Materials and methods}

Specimens. Sixty-seven biopsy specimens of primary OSCC were obtained from patients undergoing surgical resection at the Department of Oral and Maxillofacial Surgery, Kanazawa University Hospital between 1989 and 2009. The patients (38 male and 29 female subjects) ranged in age from 32 to 91 years (mean age: 60 years). Informed consent for experimental use of the samples was obtained from the patients according to the hospital's ethical guidelines.

Staining methods. Immunohistochemical staining was performed by the labeled streptavidin-biotin (LSAB) method after deparaffinization and rehydration as described by Nozaki et al (25). The sections were reacted with the following primary antibodies: anti-claudin-7 monoclonal antibody (Invitrogen Corp., Camarillo, CA, USA) diluted 200-fold with PBS at $4^{\circ} \mathrm{C}$ overnight. Sections were then reacted with a secondary antibody, biotin-labeled goat anti-rabbit immunoglobulin polyclonal antibody (Dako Japan, Kyoto, Japan) at RT for $60 \mathrm{~min}$. A section of normal oral epithelium previously identified as strong staining was used as a positive control with each batch. As a negative control, PBS treated sections instead of claudin-7 antibody was used.

Cell culture and cell lines. All cell lines were maintained at $37^{\circ} \mathrm{C}$ in a humidified incubator containing $5 \% \mathrm{CO}_{2}$. The OSCC cell lines HSC-4, OSC-20, OSC-19, OTC-04, HOC313 and TSU were maintained in minimal essential medium (MEM; SigmaAldrich, Ayrshire, UK) supplemented with $10 \%$ fetal bovine serum and $1 \%$ penicillin-streptomycin. The cell lines were derived from OSCC with the following grades of invasiveness, according to the Yamamoto-Kohama criteria (20): HSC-4 and OSC-20 cells from grade 3 as described for the low invasive type; OSC-19 and OTC-04 from grade 4C, as described for the mild invasive type; HOC313 and TSU from 4D as described for the high invasive types.

RNA extraction and reverse transcription-polymerase chain reaction $(R T-P C R)$. RT-PCR analysis was performed using a modified method by Conboy et al (26). RNA was extracted from cultured cells using an RNeasy kit (Qiagen, Hilden, Germany). A $1-\mu \mathrm{g}$ sample in $10 \mu \mathrm{l}$ of RNase free water was incubated for $5 \mathrm{~min}$ at $60^{\circ} \mathrm{C}$ and then quickly chilled on ice for $5 \mathrm{~min}$. The RNA samples were reversed-transcribed into first-strand cDNA at $40^{\circ} \mathrm{C}$ for $40 \mathrm{~min}$ in RT solution from the RNeasy kit. The cDNA samples were amplified following addition of the PCR mixture solution and the following primers for claudin-7, 5'-aat gta cga ctc ggt gct cg-3' (forward) and 5'-att ccc agg aca gga aca gg-3' (reverse); for E-cadherin, 5'-agc cat ggg cec ttg gag-3' (forward) and 5'-cca gag get ctg tca cct tc-3' (reverse); for Snail, 5'-acc act atg ccg cgc tct ttc ctc g-3' (forward) and 5'-gac agg aga agg gct tct cgc cag t-3' (reverse) and for $\beta$-actin, 5'-gaa aat ctg gca cca cac ctt-3' (forward) and $5^{\prime}$-ttg aag gta gtt tcg tgg at-3' (reverse). PCRs were carried out under the following conditions: $3 \mathrm{~min}$ at $94^{\circ} \mathrm{C}$, followed by cycles (30 for claudin-7, 30 for E-cadherin, 30 for Snail and
Table I. Yamamoto-Kohama classification.

\begin{tabular}{ll}
\hline Grade & \multicolumn{1}{c}{ Histologic grading } \\
\hline 1 & Well-defined borderline \\
2 & Cords, less marked borderline \\
3 & Groups of cells, no distinct borderline \\
$4 \mathrm{C}$ & Diffuse invasion, Cord-like type \\
$4 \mathrm{D}$ & Diffuse invasion, Widespread type \\
\hline
\end{tabular}

20 for $\beta$-actin) of $1 \mathrm{~min}$ at $94^{\circ} \mathrm{C}, 1 \mathrm{~min}$ at $58^{\circ} \mathrm{C}$, and $1 \mathrm{~min}$ at $72^{\circ} \mathrm{C}$. All reactions were completed with a final incubation at $72^{\circ} \mathrm{C}$ for $10 \mathrm{~min}$. The lengths for the amplified fragments for claudin-7, E-cadherin, Snail and $\beta$-actin genes were 288, 544, 637 and 592 bp, respectively. PCR products were detected by $3.0 \%$ agarose gel electrophoresis and staining with ethidium bromide.

Western blot analysis. Cultured cells on $80 \%$ confluent plates were used for protein samples. The protein samples $(30 \mu \mathrm{g})$ that were extracted from the whole cellular structure using M-PER (Mammalian protein extraction reagent) (Pierce, Rockford, IL, USA) were heated at $95^{\circ} \mathrm{C}$ for $5 \mathrm{~min}$ before electrophoresis and then subjected to $10 \%$ SDS-PAGE. After electrophoresis, the samples were transferred onto PVDF membranes (ATTO Co., Tokyo, Japan) and incubated for $1 \mathrm{~h}$ with 200-fold diluted polyclonal anti-rabbit antibody against claudin-7 (Invitrogen, Carlsbad, CA, USA), a 2,000-fold diluted polyclonal anti-mouse antibody against E-cadherin (BD Biosciences, San Jose, CA, USA), a polyclonal antirabbit antibody against Snail (Abgent, San-Diego, CA, USA) and 5000-fold diluted polyclonal anti-mouse antibody $\beta$-actin (Sigma, St. Louis, MO, USA) respectively. The membrane was washed three times with PBS and then incubated for $1 \mathrm{~h}$ with 2000-fold diluted horseradish peroxidase-conjugated anti-rabbit IgG (Amersham, Buckinghamshire, UK) to detect claudin-7 and Snail, 2000-fold diluted horseradish peroxidase-conjugated anti-mouse IgG (Amersham) to detect E-cadherin and $\beta$-actin, respectively. The blots were revealed by enhanced chemiluminescent detection carried out according to the manufacturer's recommendations.

Assessment of immunohistochemical staining of claudin-7 proteins. Statistical analysis was performed with the SPSS for window version 16.0 (SPSS Inc., Chicago, IL, USA). The expression of claudin-7 in tumor cells was evaluated as present or absent. Only cases in which at least $25 \%$ of the tumor cells were immunoreactive were scored as positive. The Mann-Whitney's $U$ test and $\chi^{2}$ test were used to analyze the association of claudin-7 expression with clinicopathological factors. Survival rates of claudin-7 -positive and -negative patients were calculated by the Kaplan-Meyer method, and examined for statistical significance using the log-rank test. Differences were considered significant at $\mathrm{p}$-values of $<0.05$. Uni- and multi-variate analyses for the 5-year overall survival of individual parameters were performed. 

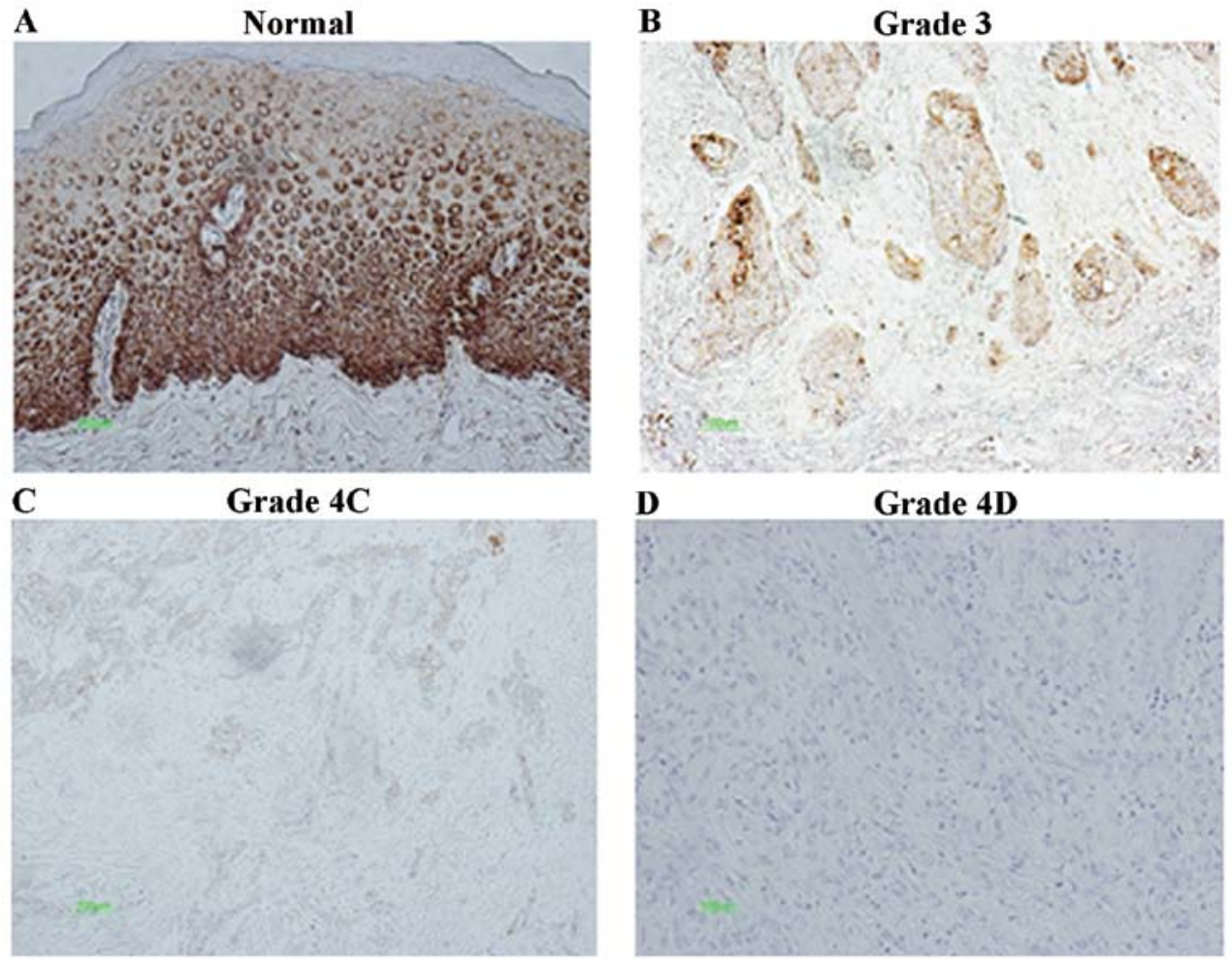

Figure 1. Immunohistochemical reactivity for claudin-7. (A) Strong expression of claudin-7 in normal tongue tissue as the positive control. (B) Mild expression of claudin-7 in grade 3 carcinoma as the positive assessment. (C) Weak expression of claudin-7 in grade $4 \mathrm{C}$ as the negative assessment. (D) No expression of claudin-7 in grade 4D as the negative assessment. Magnification, original x100.

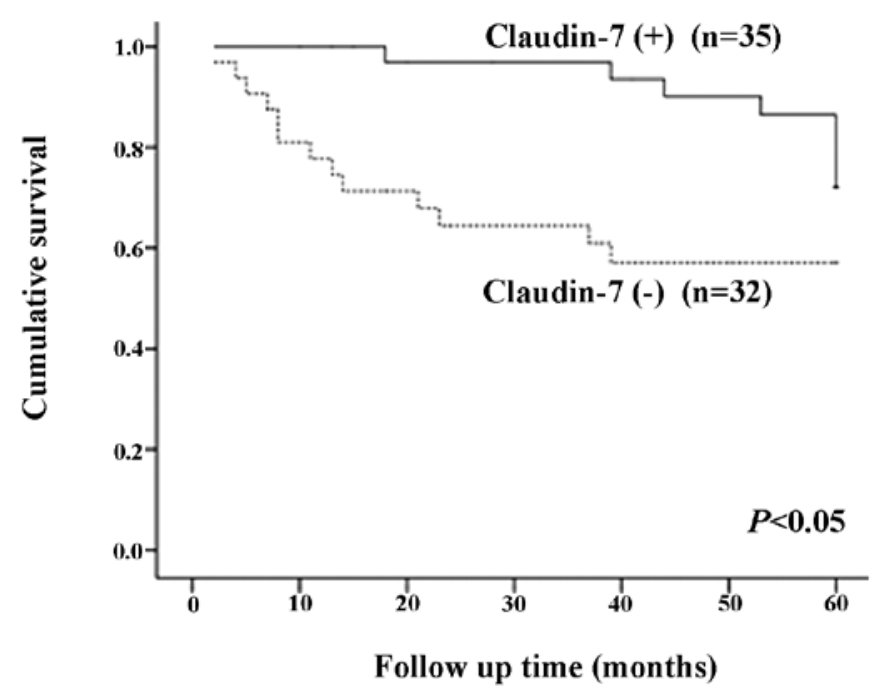

Figure 2. Kaplan-Meier survival estimates for overall survival based on claudin-7 expression $(\mathrm{P}<0.05)$.

\section{Results}

Immunohistochemistry and evaluation. The relationship between the clinicopathological parameters and expression of claudin-7 is summarized in Table II. Claudin-7 immunostaining was observed especially in membranes including the cytoplasm and nucleus of tumor cells (Fig. 1). Immunohistochemical staining showed that 35 specimens $(52.2 \%)$ were positive for claudin-7. There was a significant negative correlation between claudin-7 and T-category $(\mathrm{p}<0.05)$, Lymph node metastasis $(\mathrm{p}<0.01)$ and mode of invasion $(\mathrm{p}<0.001)$; the number of claudin-7 positive cases were $11(91.7 \%)$ for grade $1,12(75.0 \%)$ for grade 2, $9(52.9 \%)$ for grade 3, $3(18.8 \%)$ for grade $4 \mathrm{C}$, and 0 $(0 \%)$ for grade $4 \mathrm{D}$. Moreover, the number of claudin-7-positive cases was $5(26.3 \%)$ with lymph node metastasis and $30(62.5 \%)$ without lymph node metastasis. Therefore, claudin-7 expression showed a negative correlation with lymph node metastasis $(\mathrm{p}<0.01)$. The overall 5 year-survival rate was $77.1 \%$ in patients showing claudin-7 expression and $59.4 \%$ in patients without claudin-7 expression $(\mathrm{P}<0.05)$ (Fig. 2). Table III summarizes the univariate and multivariate analyses of the correlation between the clinicopathological and immunohistochemical variables, with respect to overall survival. Multivariate analysis revealed that only the 3-4D mode of invasion was significant and independent variables with relative risks of 7.44, although univariate analysis revealed that T-category, N-category, Cell differentiation, mode of invasion, expression of claudin-7 were also significant variables.

Analysis of claudin-7 mRNA, Snail and E-cadherin levels in OSCC cell lines by RT-PCR. We further examined the levels of claudin-7 mRNAs, E-cadherin and Snail in six cell lines by RT-PCR. Expressions of claudin-7 and E-cadherin were significantly lower in the HOC313 cells and TSU cells (grade 4D) while expression of Snail was higher in grade 4D than in the other cell lines (Fig. 3).

Analysis of claudin-7, Snail, E-cadherin protein levels in OSCC cell lines by western blotting. The expression of claudin-7 and E-cadherin protein was significantly lower in the HOC313 and 


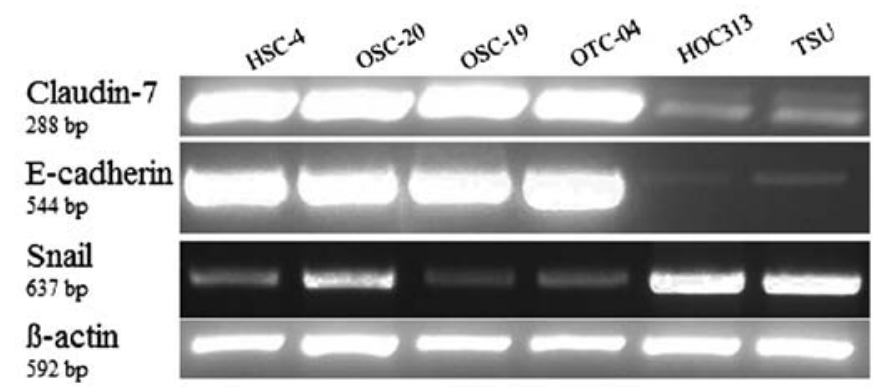

Figure 3. Expressions of mRNA for, claudin-7, E-cadherin, Snail, $\beta$-actin in six cell lines. Aliquots $(1 \mu \mathrm{g})$ of total RNAs were analyzed by reverse transcriptase-polymerase chain reaction.

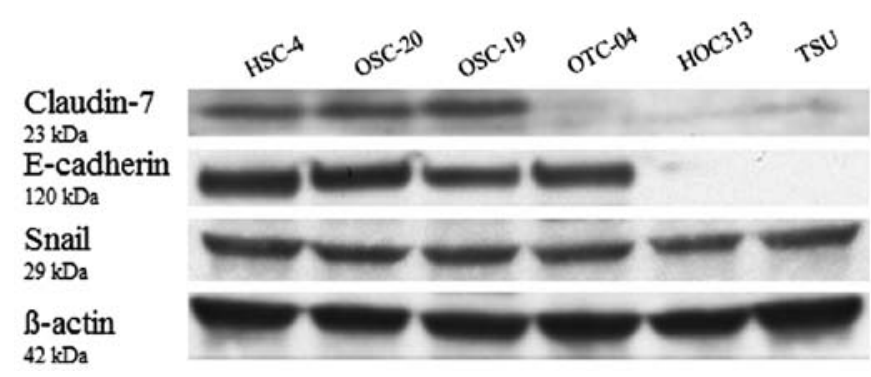

Figure 4. Expressions of protein for Claudin-7, E-cadherin, Snail, $\beta$-actin in six cell lines. The protein samples $(30 \mu \mathrm{g})$ that were extracted from the whole cellular structure were analyzed by western blotting.

TSU cell lines (grade 4D) while the expression of Snail was approximately consistent with the other cell lines (Fig. 4).

\section{Discussion}

Oral squamous cell carcinoma is characterized by a high degree of invasion into local tissue, as well as high incidence of lymph node metastasis. However, there have been few reports on the association between the expression of claudin-7 and the invasive potential in OSCC. In this study, multivariate analysis revealed that only mode of invasion in 3-4D was significant. In addition, independent variables with relative risks of 7.44 were significant variables. However, univariate analysis revealed that T-category, $\mathrm{N}$-category, cell differentiation, mode of invasion, and expression of claudin-7 were also significant. Even if limited to head and neck squamous cell carcinoma, how the pattern of claudin-7 expression correlates with invasion and metastasis is controversial. For example, Lourenço et al (1) reported that loss of claudin-7 is associated with the pathogenesis and a poor prognosis. On the other hand Bello et al (27) stated that medium immunoreactivity of claudin-7 tends to be associated with improved survival compared with strong and low immunoreactivity. This study showed that as the invasiveness of OSCC increased, the expression of claudin-7 became weaker, while studies on Snail, which reported on the suppression of E-cadherin and claudin, showed stronger expression in the most invasive mode type $4 \mathrm{D}$, which has the characteristics of EMT such as spindly shape and decreased expression of E-cadherin $(23,28)$.

Epithelial-mesenchymal transition (EMT) is one of the mechanisms by which epithelial cells acquire the motile
Table II. Clinicopathological parameters in relation to claudin-7 expression.

\begin{tabular}{lccc}
\hline Parameter & Positive (\%) & Negative (\%) & Total \\
\hline Age, years & & & \\
$\geq 65$ & $11(47.8)$ & $12(52.2)$ & 23 \\
$<65$ & $24(54.5)$ & $20(45.5)$ & 44 \\
Gender & & & \\
Male & $20(52.6)$ & $18(47.4)$ & 38 \\
Female & $15(51.7)$ & $14(48.3)$ & 29 \\
Tumor site & & & \\
Tongue & $20(51.3)$ & $19(49.7)$ & 39 \\
Gingiva & $6(40.0)$ & $9(60.0)$ & 15 \\
Oral floor & $4(80.0)$ & $1(20.0)$ & 5 \\
Buccal & $5(71.4)$ & $2(28.6)$ & 7 \\
Lip & $0(0.0)$ & $1(100.0)$ & 1 \\
T-category & & & \\
T1 & $15(75.0)$ & $5(25.0)$ & $20^{\mathrm{a}}$ \\
T2 & $16(48.5)$ & $17(51.5)$ & $33^{\mathrm{a}}$ \\
T3 & $1(25.0)$ & $4(75.0)$ & $5^{\mathrm{a}}$ \\
T4 & $3(33.3)$ & $6(66.7)$ & $9^{\mathrm{a}}$ \\
Lymph node metastasis & & & \\
Negative & $30(62.5)$ & $18(37.5)$ & $48^{\mathrm{b}}$ \\
Positive & $5(26.3)$ & $14(73.7)$ & $19^{\mathrm{b}}$ \\
Differential type & & & \\
Well & $25(58.1)$ & $18(41.9)$ & 43 \\
Moderately & $9(50.0)$ & $9(50.0)$ & 18 \\
Poorly & $1(16.7)$ & $5(83.3)$ & 6 \\
Mode of invasion & & & \\
1 & $11(91.7)$ & $1(8.3)$ & $12^{\mathrm{c}}$ \\
2 & $12(75.0)$ & $4(25.0)$ & $16^{\mathrm{c}}$ \\
3 & $9(52.9)$ & $8(47.1)$ & $17^{\mathrm{c}}$ \\
$4 \mathrm{C}$ & $3(18.8)$ & $13(81.2)$ & $16^{\mathrm{c}}$ \\
$4 \mathrm{D}$ & $0(0.0)$ & $6(100.0)$ & $6^{\mathrm{c}}$ \\
Total & $35(52.2)$ & $32(47.8)$ & 67 \\
\hline & & & \\
\hline & & & \\
\hline
\end{tabular}

${ }^{\mathrm{a} p}<0.05,{ }^{\mathrm{b}} \mathrm{p}<0.01,{ }^{\mathrm{c}} \mathrm{p}<0.001$.

properties required for invasion. Acquisition of the mesenchymal state, with fibroblastic phenotype, is accompanied by E-cadherin downregulation and upregulation of mesenchymal markers such as Snail, enabling cells to dissociate from the epithelial tissue and migrate (29). An inverse correlation between Snail and E-cadherin expression has been reported in many types of cancers including squamous cell carcinoma (30). Snail binds to E-boxes present in the E-cadherin promoter, consequently repressing E-cadherin transcription (28-31). In addition, induction of Snail expression causes loss of TJ integral membrane proteins such as claudin by a similar mechanism $(6,31)$. Accordingly, Snail may functions as an effector for EMT, and it enhances the invasive capacity of squamous cell carcinoma through the 
Table III. Univariate and multivariate analyses for clinical parameters, claudin-7 expression in relation to overall survival of 67 patients with oral squamous cell carcinoma.

\begin{tabular}{|c|c|c|c|c|c|c|c|}
\hline \multirow[b]{2}{*}{ Variables } & \multirow[b]{2}{*}{ Clinical groups } & \multirow{2}{*}{$\begin{array}{l}\text { Survivors } \\
\qquad \mathrm{n}=43\end{array}$} & \multirow{2}{*}{$\begin{array}{l}\text { Non-survivors } \\
\qquad n=24\end{array}$} & \multicolumn{2}{|c|}{ Log rank } & \multirow{2}{*}{$\begin{array}{l}\text { Cox regression } \\
\text { p-value }\end{array}$} & \multirow[b]{2}{*}{ Risk ratio $(95 \% \mathrm{CI})$} \\
\hline & & & & $\chi^{2}$ & $\mathrm{p}$-value & & \\
\hline T category & $\mathrm{T} 3,4 / \mathrm{T} 1,2$ & $5 / 38$ & $9 / 15$ & 10.48 & 0.0012 & NS & NS \\
\hline $\mathrm{N}$ category & $\mathrm{N}^{+} / \mathrm{N} 0$ & $8 / 35$ & $11 / 13$ & 8.79 & 0.0030 & NS & NS \\
\hline Cell differentiation & Mod-poor/Well & $12 / 31$ & $12 / 12$ & 5.63 & 0.018 & NS & NS \\
\hline Mode of invasion & $3-4 \mathrm{D} / 1-2$ & $18 / 25$ & $21 / 3$ & 14.46 & 0.0001 & 0.0012 & $7.44(2.221-25.06)$ \\
\hline Claudin-7 & $+/-$ & $27 / 16$ & $8 / 16$ & 6.67 & 0.0098 & NS & NS \\
\hline
\end{tabular}

NS, not significant.

regulation of proteolytic enzymes, including claudin-7 in the course of EMT.

It is difficult to determine the difference between the grade 4C type and the grade 4D type. As such, the diagnostic criteria are based solely on histopathological findings and this has created unevenness in judgments between institutions in Japan. Diagnosis may be facilitated by applying the expression of adhesion targets such as claudin-7 and E-cadherin, and the expression of Snail to discriminate grade $4 \mathrm{C}$ from grade 4D. Moreover, new identification criteria of grade 4D that include evaluation of the property of adhesion and EMT may enhance the precision of the YK criteria as a prognosis marker and contribute beneficially to the development of strategies for OSCC treatment.

In conclusion, claudin-7 may be a useful marker to identify the potential for progression with a central focus on invasion in OSCC. It is necessary to clarify the mechanism between claudin-7 expression and the process of malignant progression of OSCC though continued research and its clinical application.

\section{Acknowledgements}

We would like to thank all the members of our department for their helpful suggestions and support. This study was supported by a Grant-in-Aid for Scientific Research (no. 24792194) from the Ministry of Education, Science, Sports and Culture of Japan.

\section{References}

1. Lourenço SV, Coutinho-Camillo CM, Buim ME, et al: Claudin-7 down-regulation is an important feature in oral squamous cell carcinoma. Histopathology 57: 689-698, 2010.

2. Lu Z, Ding L, Hong H, Hoggard J, Lu Q and Chen YH: Claudin-7 inhibits human lung cancer cell migration and invasion through ERK/MAPK signaling pathway. Exp Cell Res 317: 1935-1946, 2011.

3. Lioni M, Brafford P, Andl C, et al: Dysregulation of claudin-7 leads to loss of E-cadherin expression and the increased invasion of esophageal squamous cell carcinoma cells. Am J Pathol 170: 709-721, 2007.

4. Turksen K and Troy TC: Junctions gone bad: claudins and loss of the barrier in cancer. Biochim Biophys Acta 1816: 73-79, 2011.

5. Cereijido M, Contreras RG, Shoshani L, Flores-Benitez D and Larre I: Tight junction and polarity interaction in the transporting epithelial phenotype. Biochim Biophys Acta 1778: 770-793, 2008.
6. Tsukita S, Yamazaki Y, Katsuno T and Tamura A: Tight junctionbased epithelial microenvironment and cell proliferation. Oncogene 27: 6930-6938, 2008.

7. Martin TA and Jiang WG: Loss of tight junction barrier function and its role in cancer metastasis. Biochim Biophys Acta 1788: 872-891, 2009.

8. Marchiando AM, Graham WV and Turner JR: Epithelial barriers in homeostasis and disease. Annu Rev Pathol 5: 119-144, 2010.

9. Martin TA and Jiang WG: Tight junctions and their role in cancer metastasis. Histol Histopathol 16: 1183-1195, 2001.

10. Mullin JM, Agostino N, Rendon-Huerta E and Thornton JJ: Keynote review: epithelial and endothelial barriers in human disease. Drug Discov Today 10: 395-408, 2005.

11. Usami Y, Chiba H, Nakayama F, et al: Reduced expression of claudin-7 correlates with invasion and metastasis in squamous cell carcinoma of the esophagus. Hum Pathol 37: 569-577, 2006.

12. Bornholdt J, Friis S, Godiksen S, et al: The level of claudin-7 is reduced as an early event in colorectal carcinogenesis. BMC Cancer 11: 65, 2011.

13. Hsueh C, Chang YS, Tseng NM, et al: Expression pattern and prognostic significance of claudins 1,4 , and 7 in nasopharyngeal carcinoma. Hum Pathol 41: 944-950, 2010.

14. Oshima T, Kunisaki C, Yoshihara K, et al: Reduced expression of the claudin-7 gene correlates with venous invasion and liver metastasis in colorectal cancer. Oncol Rep 19: 953-959, 2008.

15. Kim CJ, Lee JW, Choi JJ, et al: High claudin-7 expression is associated with a poor response to platinum-based chemotherapy in epithelial ovarian carcinoma. Eur J Cancer 47: 918-925, 2011.

16. Bernardi MA, Logullo AF, Pasini FS, et al: Prognostic significance of CD24 and claudin-7 immunoexpression in ductal invasive breast cancer. Oncol Rep 27: 28-38, 2012.

17. Park JY, Park KH, Oh TY, et al: Up-regulated claudin 7 expression in intestinal-type gastric carcinoma. Oncol Rep 18: 377-382, 2007.

18. Dahiya N, Becker KG, Wood WH, Zhang Y and Morin PJ: Claudin-7 is frequently overexpressed in ovarian cancer and promotes invasion. PLoS One 6: e22119, 2011.

19. Cancer UICl: TNM classification of malignant tumors, 5th edition. Wiley-Less, New York, 1997.

20. Yamamoto E, Kohama G, Sunakawa H, Iwai M and Hiratsuka H: Mode of invasion, bleomycin sensitivity, and clinical course in squamous cell carcinoma of the oral cavity. Cancer 51: 2175-2180, 1983.

21. Yoshizawa K, Nozaki S, Kitahara H, et al: Copper efflux transporter (ATP7B) contributes to the acquisition of cisplatinresistance in human oral squamous cell lines. Oncol Rep 18: 987-991, 2007.

22. Yoshizawa K, Nozaki S, Okamune A, et al: Loss of maspin is a negative prognostic factor for invasion and metastasis in oral squamous cell carcinoma. J Oral Pathol Med 38: 535-539, 2009.

23. Taki M, Kamata N, Yokoyama K, Fujimoto R, Tsutsumi S and Nagayama M: Down-regulation of Wnt-4 and up-regulation of Wnt-5a expression by epithelial-mesenchymal transition in human squamous carcinoma cells. Cancer Sci 94: 593-597, 2003.

24. Kawashiri S, Kumagai S, Kojima K, Harada H and Yamamoto E: Development of a new invasion and metastasis model of human oral squamous cell carcinomas. Eur J Cancer B Oral Oncol 31B: 216-221, 1995. 
25. Nozaki S, Endo Y, Kawashiri S, et al: Immunohistochemical localization of a urokinase-type plasminogen activator system in squamous cell carcinoma of the oral cavity: association with mode of invasion and lymph node metastasis. Oral Oncol 34: 58-62, 1998.

26. Conboy JG, Chan J, Mohandas N and Kan YW: Multiple protein 4.1 isoforms produced by alternative splicing in human erythroid cells. Proc Natl Acad Sci USA 85: 9062-9065, 1988.

27. Bello IO, Vilen ST, Niinimaa A, Kantola S, Soini Y and Salo T: Expression of claudins 1, 4, 5, and 7 and occludin, and relationship with prognosis in squamous cell carcinoma of the tongue. Hum Pathol 39: 1212-1220, 2008.

28. Ikenouchi J, Matsuda M, Furuse M and Tsukita S: Regulation of tight junctions during the epithelium-mesenchyme transition: direct repression of the gene expression of claudins/occludin by Snail. J Cell Sci 116: 1959-1967, 2003.
29. Zhu LF, Hu Y, Yang CC, et al: Snail overexpression induces an epithelial to mesenchymal transition and cancer stem cell-like properties in SCC9 cells. Lab Invest 92: 744-752, 2012.

30. Mikami S, Katsube K, Oya M, et al: Expression of Snail and Slug in renal cell carcinoma: E-cadherin repressor Snail is associated with cancer invasion and prognosis. Lab Invest 91: 1443-1458, 2011.

31. Usami Y, Satake S, Nakayama F, et al: Snail-associated epithelial-mesenchymal transition promotes oesophageal squamous cell carcinoma motility and progression. J Pathol 215: 330-339, 2008. 